\title{
Coronary Artery Bypass Grafting with Complete Revascularization Can Provide Equivalent Long-Term Prevention of Ischemic Cardiac Events in Patients on and Not on Hemodialysis: A Graft-Matched and Baseline-Adjusted Analysis
}

\author{
Hajime Imura ${ }^{1 *}$, Makoto Shirakawa ${ }^{1}$, Motohiro Maeda', Kenichiro Takahashi' ${ }^{2}$, Takahide Yosio ${ }^{2}$, \\ Yuji Maruyama1, Takashi Nitta²
}

${ }^{1}$ Department of Cardiovascular Surgery, Nippon Medical School Musashikosugi Hospital, Kanagawa, Japan

${ }^{2}$ Nippon Medical School Hospital, Kawasaki, Japan

Email: *himura@nms.ac.jp

How to cite this paper: Imura, H., Shirakawa, M., Maeda, M., Takahashi, K., Yosio, T., Maruyama, Y. and Nitta, T. (2018) Coronary Artery Bypass Grafting with Complete Revascularization Can Provide Equivalent Long-Term Prevention of Ischemic Cardiac Events in Patients on and Not on Hemodialysis: A Graft-Matched and Baseline-Adjusted Analysis. Surgical Science, 9, 367-380.

https://doi.org/10.4236/ss.2018.910044

Received: September 14, 2018

Accepted: October 28, 2018

Published: October 31, 2018

Copyright (c) 2018 by authors and Scientific Research Publishing Inc. This work is licensed under the Creative Commons Attribution International License (CC BY 4.0).

http://creativecommons.org/licenses/by/4.0/ (c) (i) Open Access

\section{Abstract}

Background: Patients on hemodialysis are at high risk of adverse cardiac events after coronary artery bypass grafting (CABG). The aim of this study is to know whether CABG with complete revascularization and similar graft selection can provide equivalent long-term benefits for patients on and not on hemodialysis. Methods: Between 2004 and 2018, 746 patients underwent isolated CABG, of which 106 were on hemodialysis. Propensity matching on baseline characteristics, graft types and on/off-pump CABG was performed to compare clinical outcomes between patients on $(\mathrm{n}=102)$ and not on $(\mathrm{n}=$ 102) hemodialysis. Results: Complete revascularization was achieved in all patients. The mean follow-up was $112.5 \pm 46.6$ months. Off-pump rates (hemodialysis vs non-hemodialysis, $93 / 102$ vs $94 / 102, p>0.999)$ and graft selections (distal anastomoses: $3.7 \pm 1.4$ vs $3.8 \pm 1.5, \mathrm{p}=0.377$, ITA grafts: $1.4 \pm$ 0.6 vs $1.5 \pm 0.6, p=0.560$, arterial grafts: $1.9 \pm 0.9$ vs $2.0 \pm 0.8, p=0.658$, vein grafts: $0.69 \pm 0.63$ vs $0.65 \pm 0.70, p=0.513$ ) were well balanced between the groups. Hospital mortality was higher in patients on hemodialysis than in those not on hemodialysis (5/102 vs $1 / 102, p=0.212)$. The Kaplan-Meier analysis revealed that cardiac death (without non-cardiac causes) was significantly more common in patients on hemodialysis than in those not on hemodialysis $(\mathrm{p}=0.018)$. However, there were no significant differences in deaths due to ischemic heart disease $(\mathrm{p}=0.327)$, repeated revascularization $(\mathrm{p}=$ 
0.542), myocardial infarction ( $\mathrm{p}=0.783)$, and heart failure requiring admission $(\mathrm{p}=0.371)$. Conclusion: CABG with complete revascularization and similar graft selection provides equivalent long-term benefits with regard to the prevention of adverse cardiac events due to ischemic heart disease in patients on and not on hemodialysis.

\section{Keywords}

Coronary Artery Bypass Grafting, Hemodialysis, Complete Revascularization, Gastroepiploic Artery

\section{Introduction}

Life expectancy is lower among patients on hemodialysis than among people without kidney disease, and approximately half of the patients on hemodialysis die from cardiovascular diseases [1]. As coronary artery disease is common and is already advanced when patients start hemodialysis [2], coronary revascularization is frequently necessary in these patients. Thus, coronary artery bypass grafting (CABG) plays an important role in maintaining the life of patients on hemodialysis.

Numerous studies have demonstrated a high incidence of adverse cardiac events in the early stage and in the long-term after CABG among patients on hemodialysis [3] [4]. Although CABG is a well-established treatment for ischemic heart disease, even in patients on hemodialysis [1] [5], the benefit may not be as favorable as that in patients not on dialysis. Marui et al. reported on the 5 -year outcomes of CABG among patients on hemodialysis and mentioned that the rates of repeated coronary revascularization and cardiac death in these patients were about twice as high as those in patients undergoing general CABG [6] [7].

Graft selection and its design have strong impacts on long-term outcomes in CABG [8] [9]. It is generally known that available grafts are relatively limited in patients on hemodialysis [5] [10], and the use of internal thoracic artery (ITA) graft is lower in patients on hemodialysis [11]. This may result in unfavorable graft selection and incomplete revascularization in such patients. As incomplete coronary revascularization is a major cause of adverse cardiac events after CABG [12], long-term outcomes in patients on hemodialysis can be impaired. However, many previous studies did not consider the graft design, the number of distal anastomoses, and rate of incomplete revascularization for the analysis of long-term results in patients on hemodialysis.

In the present study, we positively used bilateral ITA and the gastroepiploic artery (GEA) in CABG for patients on hemodialysis and achieved complete revascularization for all of them. Propensity score matching was strictly applied on graft types as well as baseline characteristics and cardiopulmonary bypass use, and we compared early and long-term outcomes between patients on and not on 
hemodialysis. The primary aim of this study was to investigate whether CABG with complete revascularization and similar graft design provides equivalent benefits with regard to the prevention of cardiac-related adverse events in patients on and not on hemodialysis. This study will also help understand what CABG with complete revascularization can provide and what that cannot do for patients on hemodialysis and with severe coronary arterial disease.

\section{Patients and Methods}

\subsection{Study Approval and Patients}

This retrospective observational study was approved by the appropriate institutional review board, and the requirement for informed consent from each patient was waived. We performed isolated CABG in 746 patients between January 2004 and June 2018, under the same strategy for graft design as follows: 1) complete revascularization, 2) use of ITA as the most reliable graft principally to the left anterior descending artery, 3) preference of an artery graft to a vein graft, and 4) off-pump CABG whenever possible. These patients were included in this study, among which 106 were preoperatively diagnosed with end-stage renal disease and were on maintenance hemodialysis. Exclusion criteria of this study included CABGs without complete revascularization, with a history of cardiac surgery, through the left thoracotomy and for patients with malignant tumor. The baseline characteristics of the patients are shown in Table 1.

Table 1. Baseline characteristics.

\begin{tabular}{|c|c|c|c|c|}
\hline & Total & Hemodialysis (-) & Hemodialysis (+) & p value ${ }^{* 3}$ \\
\hline Patient number & 746 & 640 & 106 & \\
\hline Age & $69.1 \pm 10.6$ & $70.4 \pm 9.1$ & $63.5 \pm 11.5$ & $<0.001$ \\
\hline Octogenarian (\%) & 12.1 & 12.3 & 10.4 & 0.632 \\
\hline Male & 79.6 & 80.0 & 78.3 & 0.517 \\
\hline Emergency (\%) & 9.0 & 8.9 & 9.4 & 0.855 \\
\hline Diabetes Mellitus (\%) & 57.5 & 55.2 & 68.9 & 0.001 \\
\hline Hypertension (\%) & 85.3 & 84.7 & 85.8 & 0.374 \\
\hline Hyperlipidemia (\%) & 61.0 & 64.1 & 42.5 & $<0.001$ \\
\hline Hemodialysis (\%) & 14.2 & 0.0 & 100.0 & NA \\
\hline 3 vessel disease (\%) & 75.6 & 76.4 & 70.8 & 0.224 \\
\hline $\mathrm{LVEF}^{\star 1}<40 \%(\%)$ & 17.7 & 15.6 & 30.2 & $<0.001$ \\
\hline Peripheral arterial disease & 20.2 & 17.7 & 33.0 & $<0.001$ \\
\hline Previous stroke (\%) & 12.6 & 12.3 & 14.2 & 0.634 \\
\hline $\begin{array}{c}\text { Carotid stenosis } \geq 50 \% \%^{* 2} \\
(\%)\end{array}$ & 17.6 & 18.1 & 14.2 & 0.408 \\
\hline
\end{tabular}




\subsection{Surgical Technique}

CABG was performed through a median sternotomy, and most procedures involved an off-pump technique. In patients with unstable hemodynamic parameters, cardiopulmonary bypass was used and CABG was performed under a beating-heart condition. The ITA and GEA were harvested in a skeletonized fashion by using an ultrasonic scalpel. The left anterior descending artery was re-vascularized using the ITA in most patients, and another ITA, the GEA, and the radial artery were used for other targeted coronary arteries with stenosis $\geq 90 \%$. When the in-situ GEA was not suitable for grafting (e.g., when stenosis of the targeted coronary artery was $<90 \%$ ), it was sometimes used as a free graft. The remaining targeted coronary arteries were grafted with saphenous vein grafts (SVGs). Postoperative management was carried out according to the standard procedures for CABG at our hospital. Anti-platelet agents and beta-blockers were restarted the day following surgery. For patients on hemodialysis, continuous veno-venous hemofiltration was started on the first postoperative day and hemodialysis was restarted when the patient returned to the general ward (usually on postoperative day $2-4)$.

\subsection{Data Collection and Statistical Analysis}

The primary endpoint of this study was cardiac death in the follow-up period, which included any death during the index hospitalization. Other prespecified endpoints were myocardial infarction, repeated coronary revascularizations, heart failure requiring hospital admission, death due to ischemic heart disease, and all-cause death. The definitions in this study were in accordance with those in previous studies. In brief, death was regarded as cardiac unless clear non-cardiac causes were recognized, and death due to ischemic heart disease was defined as cardiac death, except death caused by valvular disease and sudden death without findings of myocardial ischemia or coronary events [6]. The definition of myocardial infarction was based on a new abnormal Q wave or a predefined enzymatic change [7]. Follow-up data were obtained from medical records, attending physicians, and/or direct phone calls to the patients or their households.

The differences between groups were evaluated using the Mann-Whitney $U$ test for continuous variables and Fisher's exact test for binary categorical variables. Propensity-score matching using the parameters shown in Table 2 and calendar year of surgery was performed to adjust the baseline between patients on and not on hemodialysis. In this procedure, pairs of patients on and not on hemodialysis were established using 1:1 nearest-neighbor matching with a \pm 0.05 caliper and no replacement. A standardized mean difference $>0.1$ was considered to indicate imbalance. Long-term outcomes were analyzed using the Kaplan-Meier method and log-rank test for unmatched and matched cohorts. All statistical analyses were performed using SPSS $25 \mathrm{~J}$ for Windows (IBM Corp., Armonk, NY, USA). A p-value $<0.05$ was considered statistically significant for all analyses. 
Table 2. Patients characteristics and in-hospital parameters.

\begin{tabular}{|c|c|c|c|c|}
\hline & Hemodialysis (+) & Hemodialysis (-) & $\mathrm{p}$ value & $\mathrm{SMD}^{* 7}$ \\
\hline \multicolumn{5}{|l|}{ (Preoperative parameters) } \\
\hline Patient number & 102 & 102 & & \\
\hline Age & $64.4 \pm 10.6$ & $64.0 \pm 11.3$ & 0.604 & 0.037 \\
\hline Male (\%) & 75.5 & 73.5 & 0.872 & 0.023 \\
\hline Emergency (\%) & 7.8 & 7.8 & $>0.999$ & 0.000 \\
\hline Diabetes Melitus (\%) & 68.6 & 66.7 & 0.881 & 0.043 \\
\hline Hypertension (\%) & 86.3 & 85.3 & $>0.999$ & 0.028 \\
\hline Hyperlipidemia (\%) & 44.1 & 40.2 & 0.671 & 0.081 \\
\hline Number of $C A D^{* 1}$ & $2.60 \pm 0.68$ & $2.68 \pm 0.62$ & 0.406 & 0.024 \\
\hline Serum creatinine & $7.35 \pm 2.11$ & $1.06 \pm 0.54$ & $<0.001$ & $\mathrm{NA}^{\star 6}$ \\
\hline Hemodialysis duration (years) & $6.7 \pm 6.6$ & & $\mathrm{NA}^{\star 6}$ & $\mathrm{NA}^{\star 6}$ \\
\hline Peripheral arterial disease (\%) & 32.4 & 35.3 & 0.767 & 0.063 \\
\hline 3 vessel disease (\%) & 71.6 & 75.5 & 0.422 & 0.044 \\
\hline Left main disease (\%) & 28.4 & 24.5 & 0.634 & 0.068 \\
\hline $\mathrm{LVEF}^{\star^{2}}<40 \%(\%)$ & 30.4 & 27.5 & 0.758 & 0.066 \\
\hline Previous stroke (\%) & 13.7 & 13.7 & $>0.999$ & 0.000 \\
\hline $\begin{array}{c}\text { Carotid stenosis } \geq 50 \%^{* 3}(\%) \\
\text { (Operative parameters) }\end{array}$ & 13.7 & 16.7 & 0.697 & 0.083 \\
\hline Number of distal anastomosis & $3.7 \pm 1.4$ & $3.8 \pm 1.5$ & 0.377 & 0.069 \\
\hline Number of ITA ${ }^{\star 4}$ & $1.4 \pm 0.6$ & $1.5 \pm 0.6$ & 0.560 & 0.083 \\
\hline Number of arterial grafts ${ }^{\star 5}$ & $1.9 \pm 0.9$ & $2.0 \pm 0.8$ & 0.658 & 0.068 \\
\hline Number of vein graft & $0.69 \pm 0.63$ & $0.65 \pm 0.70$ & 0.513 & 0.045 \\
\hline Bilateral ITA ${ }^{* 4}(\%)$ & 51.0 & 54.9 & 0.674 & 0.080 \\
\hline All arterial grafts ${ }^{\star 5}(\%)$ & 45.1 & 43.1 & 0.888 & 0.040 \\
\hline Use of IABP (\%) & 24.5 & 21.6 & 0.740 & 0.071 \\
\hline Use of pump (\%) & 7.8 & 7.8 & $>0.999$ & 0.000 \\
\hline
\end{tabular}

${ }^{* 1}$ : Coronary artery disease. ${ }^{* 2}$ : Left ventricular ejection fraction. ${ }^{* 3}$ : determined in preoperative carotid duplex echo. ${ }^{* 4}$ : Internal thoracic artery. ${ }^{* 5}$ : arterial grafts include the radial artery and right gastroepiploic artery. ${ }^{* 6}$ : not applicable, ${ }^{* 7}$ : Standardized mean difference.

\section{Results}

\subsection{Preoperative Assessments}

The frequencies of diabetes, low left ventricular ejection fraction, and peripheral arterial disease were greater, age was lower, and the frequency of hyperlipidemia was lower in patients on hemodialysis than in those not on hemodialysis (Table 1). Thus, baseline characteristics were significantly different between the groups before matching. As shown in Table 2, the groups were well-balanced with regard to preoperative characteristics as well as graft types and other surgical pa- 
rameters after matching.

\subsection{In-Hospital Outcomes}

Table 3 presents the in-hospital outcomes after CABG. The surgical mortality rate was higher in patients on hemodialysis than in those not on hemodialysis, but the difference was not significant $(4.90 \%$ vs. $0.98 \%, \mathrm{p}=0.212)$. However, ventilation time, intensive care unit stay, and hospital stay were significantly longer in patients on hemodialysis than in those not on hemodialysis (Table 3 ). Five deaths occurred among patients on hemodialysis, and they were related to heart failure $(n=2)$, sepsis $(n=2)$, and peripheral arterial disease $(n=1)$. Three of the five deaths occurred after uneventful recovery in the ward without cardiac-related problems.

\subsection{Long-Term Outcomes}

The mean follow-up period was $112.5 \pm 46.6$ months in the entire cohort, and there were 47 deaths after CABG (Table 4). The rate of all-cause death was significantly higher in patients on hemodialysis than in those not on hemodialysis (Figure 1).

\section{Cardiac-related death}

The incidence of cardiac death in the long-term was significantly higher in patients on hemodialysis than in those not on hemodialysis $(p=0.018$, Figure 2). Eleven cardiac deaths were observed among patients on hemodialysis (five surgical deaths, three heart failures, and three sudden deaths) (Table 4). As all these sudden deaths occurred in patients who did not show findings of myocardial ischemia, heart failure, or ventricular arrhythmia before death, the physicians in charge speculated that the deaths were associated with electrolyte disorders or cerebrovascular events. Thus, more than $50 \%$ of cardiac deaths were not associated with clear issues of the heart in patients on hemodialysis, while all four cardiac deaths were related to cardiac diseases in patients not on hemodialysis (Table 4).

\section{Death due to ischemic heart disease}

Six deaths (five surgical deaths and one death related to non-valvular heart failure in the long-term) were identified as deaths due to ischemic heart disease in patients on hemodialysis, while four such deaths (one surgical death, one death related to non-valvular heart failure, and two deaths related to ischemic mortal arrhythmia) were observed in patients not on dialysis. There was no significant difference in the event-free rate of death due to ischemic heart disease between the groups ( $\mathrm{p}=0.327$, Figure 3 ).

Repeated revascularization, myocardial infarction, and heart failure requiring admission

Figure 4 shows the event-free curves for repeated revascularization, myocardial infarction, and heart failure requiring hospital admission. Twenty patients underwent repeated coronary revascularization in this study, and all of the pa- 
tients underwent percutaneous coronary intervention (PCI). Among the 20 patients, 10 patients on hemodialysis underwent a total of $11 \mathrm{PCI}$ procedures (6 were for new lesions and 5 were for graft failure), whereas 10 patients not on hemodialysis underwent a total of 12 PCI procedures (5 were for new lesions and 7 were for graft failure). There was no significant difference in repeated revascularization in the long-term between the groups $(\mathrm{p}=0.571)$.

There were no significant differences in the event-free rates of myocardial infarction and heart failure requiring admission between the groups ( $\mathrm{p}=0.783$ and $\mathrm{p}=0.371$, respectively)

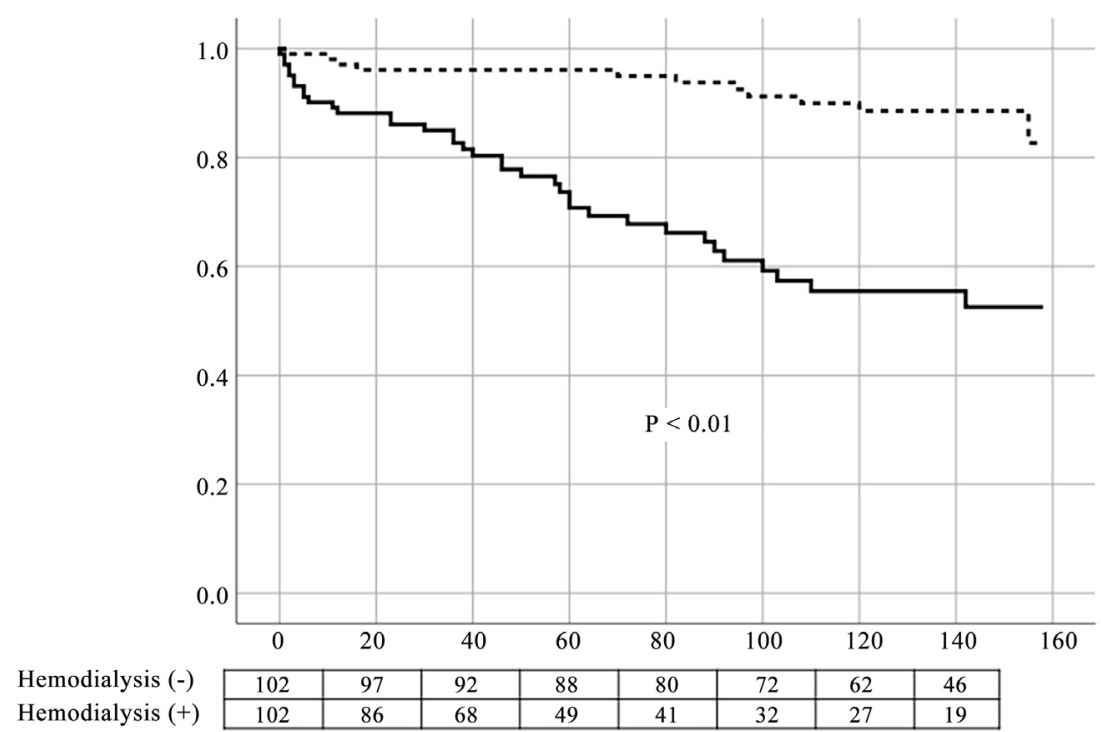

Figure 1. Survival curves of patients on hemodialysis (solid line) and those not on hemodialysis (dotted line).

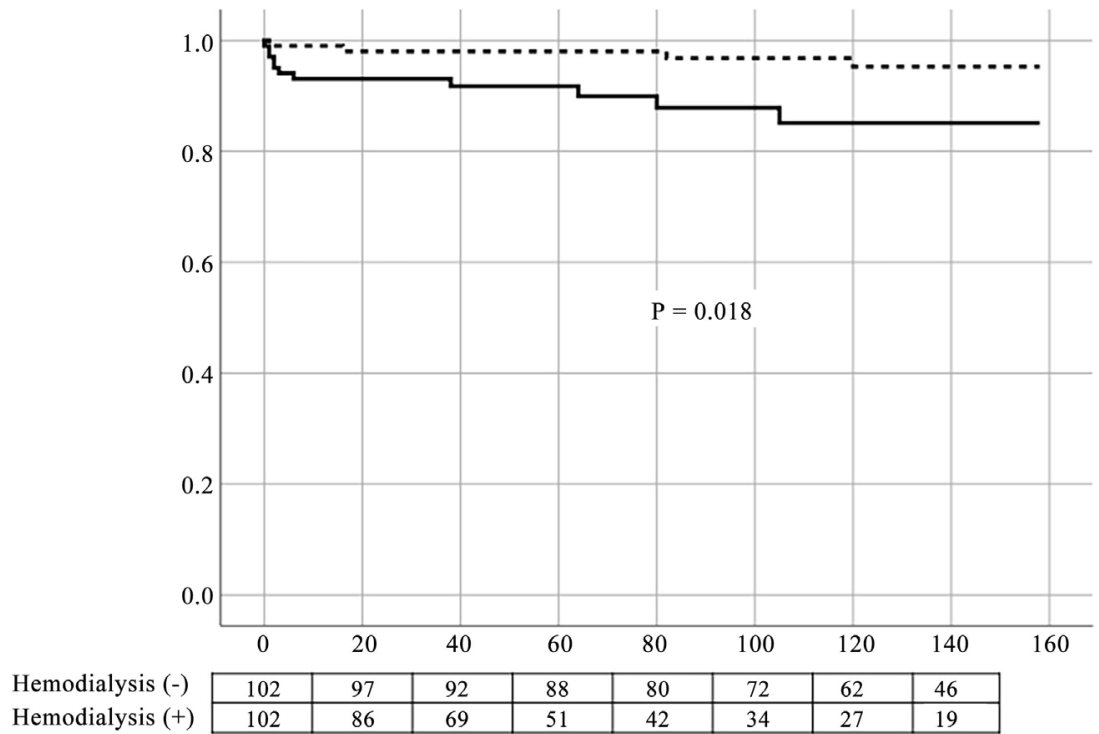

Figure 2. Freedom from cardiac death (any deaths without non-cardiac causes). Solid line shows patients on hemodialysis and dotted line shows those not on hemodialysis. 


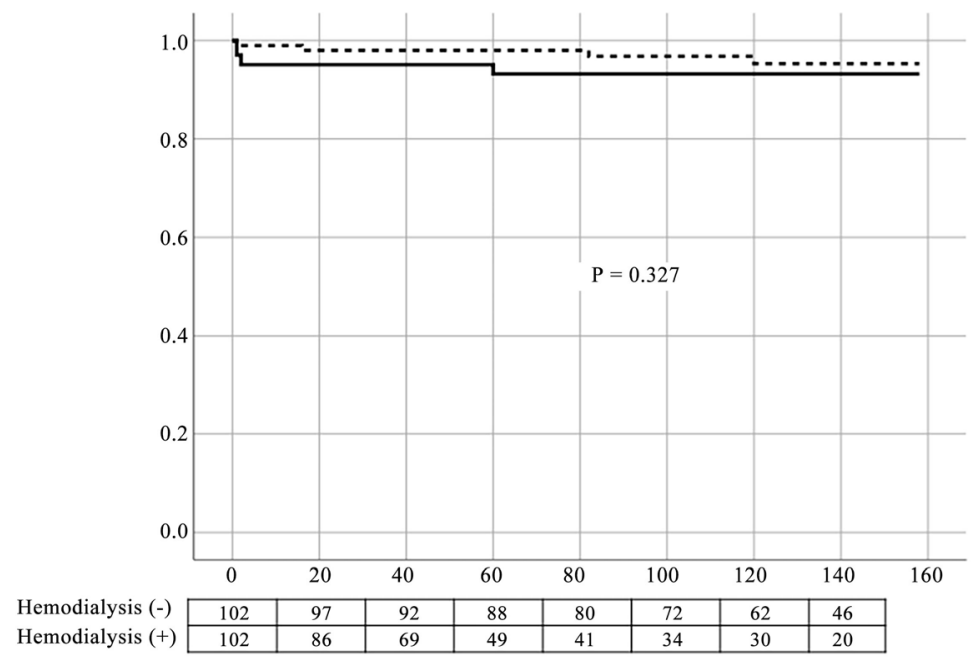

Figure 3. Freedom from death due to ischemic heart disease (cardiac death except death due to valvular disease and sudden death without findings of myocardial ischemia). Solid line shows patients on hemodialysis and dotted line shows those not on hemodialysis.

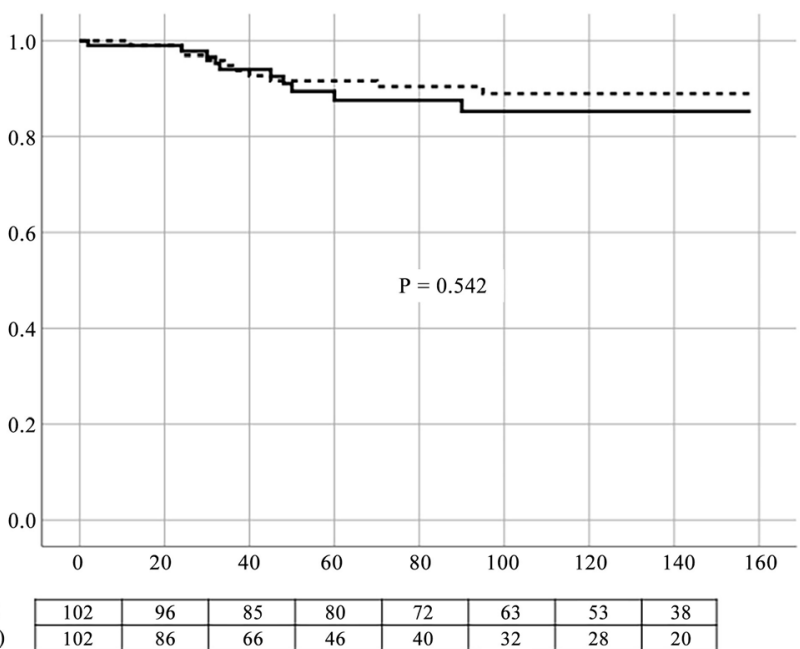

Hemodialysis (-) Hemodialysis (+)

(a)

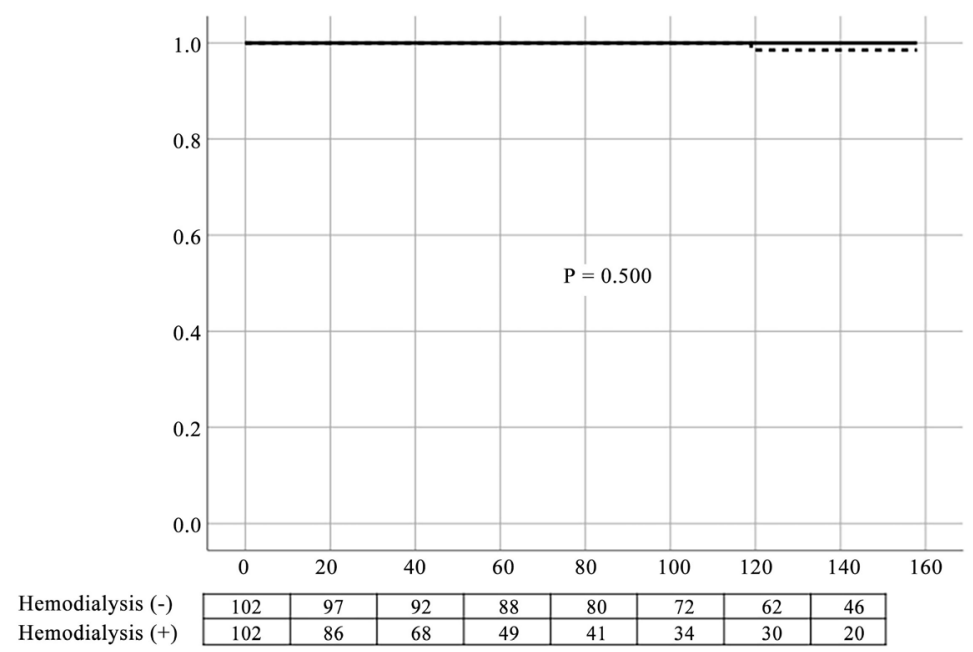

(b) 


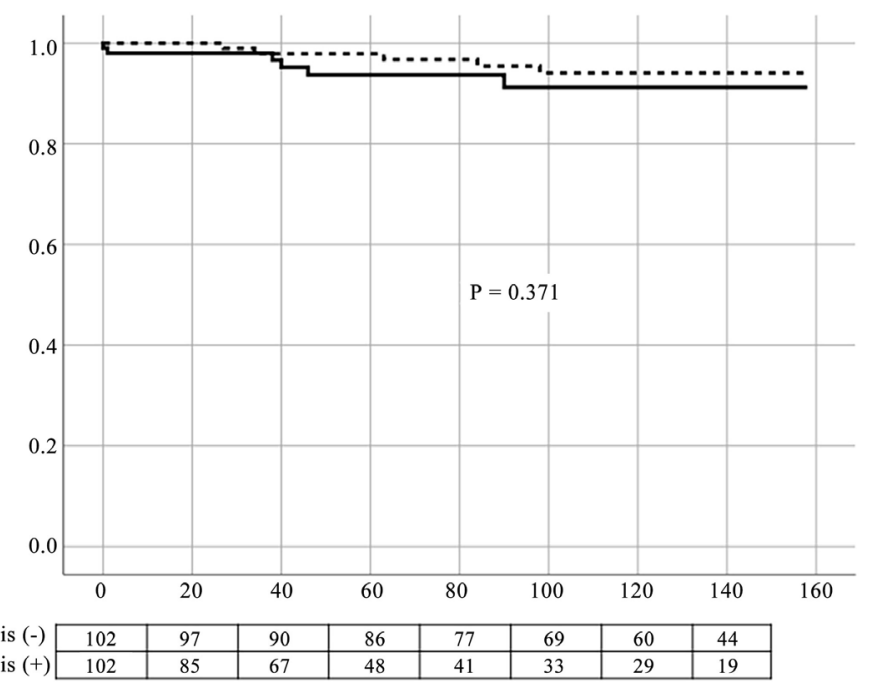

(c)

Figure 4. Freedom from repeated coronary revascularization (a), myocardial infarction (b) and heart failure requiring hospital admission (c). Solid line shows patients on hemodialysis and dotted line shows those not on hemodialysis. There were no significant differences between the two groups in each analysis.

Table 3. Postoperative course and in-hospital mortality.

\begin{tabular}{cccc}
\hline & Hemodialysis $(+)$ & Hemodialysis $(-)$ & P value \\
\hline Graft patency & 98.4 & 98.1 & 0.154 \\
Ventilation time (hours) & $19.0 \pm 32.3$ & $11.1 \pm 13.4$ & 0.003 \\
ICU ${ }^{\star 2}$ stay (days) & $4.0 \pm 3.2$ & $3.2 \pm 1.8$ & 0.052 \\
Hospital stay (days) & $25.2 \pm 113.3$ & $19.2 \pm 9.7$ & 0.001 \\
30 days mortality (\%) & 2.0 & 0.98 & $>\mathbf{0 . 9 9 9}$ \\
Hospital death (\%) & 4.90 & 0.98 & 0.212 \\
\hline
\end{tabular}

${ }^{* 1}$ : graft patency was examined for 60 patients on hemodialysis and 76 patients without hemodialysis, generally within a month from surgery by angiography or computed tomography. ${ }^{* 2}$ : Intensive care unit.

Table 4. Causes of death through the observation period.

\begin{tabular}{ccc}
\hline Cardiac Death $(\mathrm{n}=16)$ & Hemodialysis $(+),(\mathrm{n}=11)$ & Hemodialysis $(-),(\mathrm{n}=4)$ \\
\hline Ischemic heart disease & 6 & 4 \\
Myocardial infarction & 0 & 2 \\
Heart failure & 3 & 1 \\
Others & 3 & 0 \\
Sudden death & 3 & 0 \\
Valvular disease & 2 & Hemodialysis $(-),(\mathrm{n}=7)$ \\
\hline Non-cardiac Death $(\mathrm{n}=31)$ & Hemodialysis $(+),(\mathrm{n}=25)$ & 2 \\
Infection & 9 & 3 \\
Malignancy & 5 & 0 \\
Stroke & 4 & 0
\end{tabular}




\section{Discussion}

The main findings of the present study are that patients on hemodialysis have acceptable incidences of repeated coronary revascularization, myocardial infarction, and heart failure and that the incidences are not significantly different from those in patients not on hemodialysis in the long-term after CABG with complete revascularization. The high incidence of cardiac death noted in patients on hemodialysis was mainly due to not cardiac related death in the postoperative period and sudden death in the long-term without signs of heart failure and/or mortal arrhythmias, and the event-free rate of death due to ischemic heart disease was not significantly different between the study groups. For the analyses, our cohorts (on and not on hemodialysis) were strictly adjusted, and therefore, the groups were similar with regard to preoperative characteristics and CABG design. This study demonstrated that, although the rate of cardiac death in the long-term was still higher in patients on hemodialysis, CABG with complete revascularization could equally prevent adverse cardiac events due to myocardial ischemia in patients on and not on hemodialysis. To our knowledge, this is the first report to present this benefit.

A large-scale multicenter study reported the details of graft design in off-pump CABG in which the mean number of distal anastomosis was 3.2 and the percentage of each graft used was as follows: left ITA, 94\%; right ITA, 52\%; GEA, 34\%; radial artery, 23\%; and vein, 42\% [13]. Because patients not on hemodialysis were selected to match with those on hemodialysis, there was a concern that the graft design for them could be different from general CABG. However, in our study, the graft use in patients not on hemodialysis were similar to that the previous report (left ITA, 97\%; right ITA, 55\%; GEA, 32\%; radial artery, $21 \%$; and vein, $52 \%)$. The difference in the mean number of distal anastomosis (3.8 in our study) can be explained by the marginally higher use of vein graft in this study. Thus, the graft design in this study appears to reflect general CABG; therefore, the clinical outcomes were not affected by the difference of graft design.

The limitation of available grafts can be a reason for incomplete coronary revascularization, and therefore, patients on hemodialysis are at risk for this issue. For example, the radial artery is usually not available in this population and the SVG might sometimes be absent because of previous surgery for peripheral arterial disease [10]. To overcome this issue, we used the GEA in $48 \%$ of patients and successfully achieved complete revascularization among patients on hemodialysis. This graft can be used as a free graft as well as an in-situ graft, and it can increase the rate of CABG with total arterial grafts. The GEA can attach well to small and/or severely diseased coronary arteries, which are frequently seen in CABG among patients on hemodialysis. It should be noted that we did not observe any patients with major adverse events associated with GEA graft failure among patients on hemodialysis. Previous studies have shown predominance of the GEA graft over the SVG as well as the advantage of total arterial grafts over 
other grafts including the SVG [14] [15]. Thus, we believe that the GEA is a useful option in patients on hemodialysis. We always assessed the presence of atherosclerotic change in the GEA on preoperative abdominal computed tomography and did not use the artery when significant calcification was noted.

The rate of bilateral ITAs among patients on hemodialysis in this study (51.0\%) was higher than that in previous studies (usually $22.8 \%$ - 40.3\%) [10] [11] [16] and was similar to that for general CABG [13]. The ITA is known to be the most reliable graft in CABG, and in fact, repeated coronary revascularization because of ITA-graft failure was significantly less frequent than repeated coronary revascularization because of SVG failure $(1 / 149$ vs. $4 / 70, p=0.038)$ in this study. Thus, the rate of repeated revascularization in this study was lower than that in previous studies and equivalent to that for non-dialysis patients [7] [17]. Furthermore, the proportion of death due to ischemic heart disease among all deaths in this study (16.7\%) was lower than that in a multicenter registry study (37\%) [6]. Thus, our findings support the use of bilateral ITAs in patients on hemodialysis; however, some studies have shown no advantage of bilateral ITAs with regard to survival among patients on hemodialysis. Nakatsu et al. reported that bilateral ITAs were not recommended for patients at high risk with regard to survival [16], and another study showed that severe diabetes, low ejection fraction, and peripheral arterial disease were risk factors with regard to survival in patients on hemodialysis [18]. Additionally, deep sternal wound infection is a concern while using bilateral ITAs [19]. We agree with these reports and refrained from using bilateral ITA for patients at high risks of the abovementioned complications [10] [18]. This was the reason we did not use bilateral ITA for nearly a half of the patients on hemodialysis. The findings of these studies indicate the importance of patient selection for bilateral ITA use.

Previous studies demonstrated long-term survival after CABG in patients on hemodialysis, and the survival rate ranged from about $30 \%$ to $60 \%$ at 5 years and $15 \%$ to $40 \%$ at 10 years [6] [7] [16] [19] [20]. Additionally, the Japanese Society for Dialysis Therapy reported (in Japanese) that general hemodialysis patients had a survival rate of approximately $40 \%$ at 10 years and revealed that the causes were heart failure (26\%), infection (22\%), malignant tumor (10\%), cerebrovascular disease (6.5\%), myocardial infarction (4\%), uremia (4\%), hyperkalemia (2.5\%), unknown causes (11\%), and others (14\%). The survival rates in our study ( $70 \%$ and $55 \%$ at 5 and 10 years, respectively) appear to be better than the rates reported in these previous studies, and this might be explained by the low incidence of deaths due to heart failure and myocardial infarction $(13.9 \%$ and $0 \%$, respectively). These findings may justify our strategy (complete revascularization with positive use of bilateral ITA and GEA) with regard to CABG for patients on hemodialysis. On the other hand, the present study and previous studies showed that patients on hemodialysis are still at high risk for septic and sudden death in the long-term even after successful CABG with complete revascularization [7] [21]. Thus, further improvement of CABG is necessary in this population. 


\section{Study limitations}

The present study had several limitations. First, this was a retrospective observational study, and therefore, some unintentional underestimation and bias could have influenced the results, although we adopted propensity-score matching. Second, as mentioned in the discussion, the radial artery could not be used, and its absence was covered by GEA in patients on hemodialysis. There may be a difference in graft performance and durability between these two arteries; however, we did not experience graft failure or other problems in both. Finally, all patients were ethnically Japanese and were treated by a single surgical team, which might have introduced ethnic and surgeon-based biases.

\section{Conclusion}

CABG with complete revascularization and similar graft selection provides equivalent benefits with regard to the prevention of repeated revascularization, myocardial infarction, heart failure requiring admission and death due to ischemic heart disease in patients on and not on hemodialysis. Positive use of bilateral ITA and GEA helped achieve complete revascularization in patients on hemodialysis. Postoperative non-cardiac related death and sudden death without myocardial ischemia and heart failure contribute to the high rate of cardiac death in patients on maintenance hemodialysis.

\section{Acknowledgements}

The authors would like to thank Enago for the English language review.

\section{Funding Statement}

This research received no specific grant from any funding agency in the public, commercial, or not-for-profit sectors.

\section{Conflicts of Interest}

The authors declare no conflicts of interest regarding the publication of this paper.

\section{References}

[1] Reddan, D.N., Szczech, L.A., Tuttle, R.H., et al. (2003) Chronic Kidney Disease, Mortality, and Treatment Strategies among Patients with Clinically Significant Coronary Artery Disease. Journal of the American Society of Nephrology, 14, 2373-2380. https://doi.org/10.1097/01.ASN.0000083900.92829.F5

[2] Joki, N., Hase, H., Nakamura, R., et al. (1997) Onset of Coronary Artery Disease Prior to Initiation of Haemodialysis in Patients with End-Stage Renal Disease. Nephrology Dialysis Transplantation, 12, 718-723. https://doi.org/10.1093/ndt/12.4.718

[3] Hori, D., Yamaguchi, A. and Adachi, H. (2017) Coronary Artery Bypass Surgery in End-Stage Renal Disease Patients. Annals of Vascular Diseases, 10, 79-87. https://doi.org/10.3400/avd.ra.17-00024 
[4] Chang, T.I., Shilane, D., Kazi, D.S., et al. (2012) Multivessel Coronary Artery Bypass Grafting versus Percutaneous Coronary Intervention in ESRD. Journal of the American Society of Nephrology, 23, 2042-2049. https://doi.org/10.1681/ASN.2012060554

[5] Hemmelgarn, B.R., Southern, D., Culleton, B.F., et al. (2004) Survival after Coronary Revascularization among Patients with Kidney Disease. Circulation, 110, 1890-1895. https://doi.org/10.1161/01.CIR.0000143629.55725.D9

[6] Marui, A., Kimura, T., Nishiwaki, N., et al. (2014) Percutaneous Coronary Intervention versus Coronary Artery Bypass Grafting in Patients with End-Stage Renal Disease Requiring Dialysis (5-Year Outcomes of the CREDO-Kyoto PCI/CABG Registry Cohort-2). American Journal of Cardiology, 114, 555-561. https://doi.org/10.1016/j.amjcard.2014.05.034

[7] Kimura, T., Morimoto, T., Furukawa, Y., et al. (2008) Long-Term Outcomes of Coronary-Artery Bypass Graft Surgery versus Percutaneous Coronary Intervention for Multivessel Coronary Artery Disease in Bare-Metal Stent Era. Circulation, 118, s199-s209. https://doi.org/10.1161/CIRCULATIONAHA.107.735902

[8] Lytle, B.W., Blackstone, E.H., Sabik, J.F., Houghtaling, P., Loop, F.D. and Cosgrove, D.M. (2004) The Effect of Bilateral Internal Thoracic Artery Grafting on Survival during 20 Postoperative Years. The Annals of Thoracic Surgery, 78, 2012-2014. https://doi.org/10.1016/j.athoracsur.2004.05.070

[9] Shilane, D., Hlatky, M.A., Winkelmayer, W.C. and Chang, T.I. (2015) Coronary Artery Bypass Graft Type and Outcomes in Maintenance Dialysis. The Journal of Cardiovascular Surgery, 56, 463-471.

[10] Nakahara, Y., Yoshida, S., Kanemura, T., Yamagishi, S., Tochigi, S. and Osaka, S. (2018) Bilateral Internal Thoracic Artery Grafts in Hemodialysis: A Single-Center Propensity Score Analysis. The Annals of Thoracic Surgery, 105, 153-159. https://doi.org/10.1016/j.athoracsur.2017.05.084

[11] Yamauchi, T., Miyata, H., Sakaguchi, T., et al. (2012) Coronary Artery Bypass Grafting in Hemodialysis-Dependent Patients-Analysis of Japan Adult Cardiovascular Surgery Database. Circulation Journal, 76, 1115-1120. https://doi.org/10.1253/circj.CJ-11-1146

[12] Benedetto, U., Gaudino, M., Franco, D.A., et al. (2018) Incomplete Revascularization and Long-Term Survival after Coronary Artery Bypass Surgery. International Journal of Cardiology, 254, 59-63. https://doi.org/10.1016/j.ijcard.2017.08.005

[13] Marui, A., Okabayashi, H., Komiya, T., et al. (2012) Benefits of Off-Pump Coronary Artery Bypass Grafting in High-Risk Patients. Circulation, 126, s151-s157. https://doi.org/10.1161/CIRCULATIONAHA.111.083873

[14] Suma, H., Tanabe, H., Takahashi, A., et al. (2007) Twenty Years Experience with the Gastroepiploic Artery Graft for CABG. Circulation, 116, 1188-1191.

[15] Tatoulis, J. (2013) Total Arterial Coronary Revascularization-Patient Selection, Stenoses, Conduits, Targets. Annals of Cardiothoracic Surgery, 2, 499-506.

[16] Nakatsu, T., Tamura, N., Sakakibara, Y., Hagio, K. and Ishigami, M. (2010) Long-Term Survival after Coronary Arterial Grafts in Patients with End-Stage Renal Disease. The Annals of Thoracic Surgery, 90, 738-743. https://doi.org/10.1016/j.athoracsur.2010.04.069

[17] Krishnaswami, A., McCllouch, C.E., Tawadrous, M., et al. (2015) Coronary Artery Bypass Grafting and Percutaneous Coronary Intervention in Patients with End-Stage Renal Disease. European Journal of Cardio- Thoracic Surgery, 47, e193-e198. https://doi.org/10.1093/ejcts/ezv104 
[18] Takami, Y., Tajima, K., Kato, W., et al. (2012) Predictors for Early and Late Outcomes after Coronary Artery Bypass Grafting in Hemodialysis Patients. The Annals of Thoracic Surgery, 94, 1940-1946.

https://doi.org/10.1016/j.athoracsur.2012.07.037

[19] Saito, A., Miyata, H., Motomura, N., Ono, M. and Takamoto, S. (2013) Propensity-Matched Analysis of Bilateral Internal Mammary Artery vs. Single Internal Mammary Artery in 7702 Cases of Isolated Coronary Artery Bypass Grafting. European Journal of Cardio- Thoracic Surgery, 44, 711-717. https://doi.org/10.1093/ejcts/ezt157

[20] Kumada, Y., Ishii, H., Aoyama, T., et al. (2014) Long-Term Clinical Outcome after Surgical or Percutaneous Coronary Revascularization in Hemodialysis Patients. Circulation Journal, 78, 986-992. https://doi.org/10.1253/circj.CJ-13-1357

[21] Charytan, D.M. and Kuntz, R.E. (2007) Risks of Coronary Artery Bypass Surgery in Dialysis-Dependent Patients-Analysis of the 2001 National Inpatient Sample. Nephrology Dialysis Transplantation, 22, 1665-1671.

https://doi.org/10.1093/ndt/gfl835 